\section{"Então não tenho como dimensionar": um retrato de grupos educativos em saúde na cidade de São Paulo, Brasil}

\author{
"So I have no way of measuring": a portrait \\ of health education groups in the city of \\ São Paulo, Brazil
}

\section{"Entonces, no sé como dimensionar": un retrato de grupos educativos en salud en la ciudad de São Paulo, Brasil}

\section{Resumo}

O estudo analisou as representações sociais dos profissionais da saúde da atenção básica sobre os processos avaliativos de grupos que abordam alimentação e nutrição, e descreveu as estratégias educativas usadas para esse cuidado. Trata-se de uma pesquisa qualitativa, conduzida na cidade de São Paulo, Brasil, entre 2012 e 2014, em que foram analisadas 48 entrevistas. Na análise das entrevistas, para a classificação das estratégias educativas em categorias e conteúdos de aprendizagem, usaram-se Bogdan er Biklen e Zabala, respectivamente. Para os processos avaliativos, foi utilizada a técnica do Discurso do Sujeito Coletivo, fundamentada nas representações sociais de Jodelet. Verificaram-se três conteúdos de aprendizagem das estratégias educativas e quatro representações sociais dos processos avaliativos que, associados, evidenciaram a presença de um conflito entre, por um lado, uma prática direcionada, pelo processo de trabalho, para critérios de avaliação quantitativos e individuais e, por outro lado, uma prática promotora da saúde, por meio do uso de abordagens inclusivas e de avaliação participativa. Nesta prática, identificou-se, implicitamente, a presença da autonomia em saúde. Evidenciou-se a necessidade de se reconhecer e sistematizar o planejamento dos grupos como uma ferramenta educativa que qualifica e potencializa o cuidado para uma atenção integral.

Atenção Primária à Saúde; Educação em Saúde; Educação Alimentar e Nutricional; Avaliação de Processos (Cuidados de Saúde)
Kellem Regina Rosendo Vincha 1

Viviane Laudelino Vieira 1

Lúcia Dias da Silva Guerra 1

Fernanda Cangussu Botelho 1

Alexandra Pava-Cárdenas 1

Ana Maria Cervato-Mancuso 1

doi: 10.1590/0102-311X00037116

\section{Correspondência}

K. R. R. Vincha

Faculdade de Saúde Pública, Universidade de São Paulo. Av. Dr. Arnaldo 715, São Paulo, SP 01246-904, Brasil. kvincha@yahoo.com.br

1 Faculdade de Saúde Pública, Universidade de São Paulo, São Paulo, Brasil. 


\section{Introdução}

Em expansão por todo o território nacional, a Estratégia Saúde da Família (ESF) é definida por um conjunto de ações e serviços que vai além da assistência clínica, estruturada com base no reconhecimento das necessidades de saúde da população. A ESF é desenvolvida, dentro da atenção básica, sob forma de trabalho em equipe, sendo dirigida a populações de territórios definidos por meio do exercício de práticas democráticas, participativas 1 e de cuidado e gestão.

Consta, como uma das práticas da atenção básica, o atendimento em grupo, o qual tem sido incentivado pela Política Nacional de Atenção Básica 1 e pela Política Nacional de Promoção da Saúde 2, direcionadoras das ações e dos serviços desse contexto. Esse atendimento apresenta vantagens em relação ao atendimento individual, pois favorece a comunicação entre os atores, o encontro dos semelhantes para a constituição de redes sociais e a ampliação do acesso à saúde. Esse acesso diz respeito à quantidade em termos de números de atendimentos e à qualidade do cuidado na direção de uma atenção integral das condições e dos modos de vida dos usuários 3 .

A principal função do grupo, dentro da atenção básica, situa-se na educação em saúde que, compreendida em uma prática social, é construída em processos dialógicos entre usuários e profissionais da área, a fim de produzir novos conhecimentos e transformação de cuidado em saúde, a partir dos diferentes saberes 4 . Em decorrência da compreensão, o presente estudo elegeu o termo "grupo" para referir-se ao grupo educativo.

A presença frequente de grupos que abordam a alimentação e nutrição desenvolvidas por profissionais de diferentes formações é consequência do aumento das doenças crônicas relacionadas ao comportamento alimentar e da dinâmica da ESF 5,6. Além disso, atribui-se à educação alimentar e nutricional a conceituação de ser “...um campo de conhecimento e de prática contínua e permanente, transdisciplinar, intersetorial e multiprofissional” 7 (p. 23).

Os grupos como ação educativa em saúde envolvem planejamento e seleção, de forma sistematizada ou não pelos profissionais da saúde, de diversos elementos como o diagnóstico, o objetivo, a mensagem, a estratégia educativa e a avaliação ${ }^{8}$. Salienta-se que, com a expansão das equipes de ESF, o último elemento tem sido colocado em discussão para verificar a resolubilidade das ações de saúde da atenção básica 9 .

Recomenda-se que a avaliação seja realizada na rotina da gestão e dos serviços de saúde, por conceber que o olhar avaliativo deve compor os processos de trabalho. Desse modo, a construção de processos avaliativos, que considera elementos de estrutura, aspectos organizativo, técnico-científico, interpessoal e de resultado, deve estar conectada com os modos de vida da população e as realidades locais 3,9. Assim, adotou-se, aqui, o termo "processo avaliativo" para se referir à avaliação de grupos, compreendendo-o como uma análise crítica de sua realização e de seus resultados, sinalizada por critérios pautados nas estratégias educativas 3,8 .

Contudo, dado que a educação alimentar e nutricional visa a promover comportamentos alimentares saudáveis, que estão relacionados com dimensões cognitivas, afetivas e habilidades 10 , construir processos avaliativos que analisem tal intencionalidade torna-se um desafio para os profissionais da saúde, sobretudo, quando aplicados em grupos, por se transformarem em um desafio coletivo.

Ações de educação alimentar e nutricional planejadas, desenvolvidas e avaliadas com base em critérios de diferentes naturezas são necessárias para evidenciar e qualificar essa prática. Do mesmo modo, também são essenciais para que a educação alimentar e nutricional seja uma ação estratégica para a promoção da saúde, da segurança alimentar e nutricional e do direito humano à alimentação adequada 7 . Diante disso, este estudo teve por objetivo analisar as representações sociais de profissionais da atenção básica sobre os processos avaliativos de grupos que abordam alimentação e nutrição, e descrever as estratégias educativas adotadas para esse cuidado.

\section{Métodos}

Trata-se de uma pesquisa exploratória, à luz da abordagem qualitativa, na qual se adotou o Marco de Referência de Educação Alimentar e Nutricional para as Políticas Públicas 7 como referencial teórico da educação alimentar e nutricional. O estudo foi conduzido na cidade de São Paulo, Brasil, que apresen- 
ta uma estrutura organizacional de saúde composta pela Secretaria Municipal de Saúde (SMS), cinco Coordenadorias Regionais de Saúde (CRS) e 24 Supervisões Técnicas de Saúde, com gestão públicoprivada compartilhada com as Organizações Sociais de Saúde (OSS). O estudo foi realizado dentro da área técnica da atenção básica, que é composta pela equipe nuclear da ESF, equipe Núcleo de Apoio à Saúde da Família (NASF), equipe de saúde bucal e equipe do Programa Ambientes Verdes e Saudáveis.

No processo de construção do universo empírico do estudo, buscaram-se profissionais que apresentassem as condições necessárias para a explicitação da problemática de investigação e que aceitassem participar voluntariamente do estudo. Para tanto, utilizaram-se como critérios de inclusão os seguintes: ser mediador de grupo e que o grupo tivesse a temática da alimentação e nutrição na abordagem.

Como primeira etapa de seleção para a composição da população do estudo, foram identificados os nutricionistas de equipe NASF, por se compreender que esses profissionais seriam inicialmente os reconhecidos em decorrência da temática. Para a localização desse público, tiveram-se como informantes-chave membros de seis OSS, e os contatos foram conduzidos por meio da técnica de bola de neve 11, em que os primeiros entrevistados recomendaram outros que, por sua vez, recomendaram outros, e assim por diante. Estabeleceu-se para essa etapa um período de seis meses e, então, ampliouse o universo empírico para outros profissionais da atenção básica.

Na segunda etapa de seleção, a localização dos outros profissionais contou com membros das cinco CRS e gerentes das unidades básicas de saúde (UBS) como informantes-chave. As CRS indicaram as UBS e, no contato com os respectivos gerentes, eles indicaram os profissionais que potencialmente poderiam contribuir para o estudo. O tempo pré-estabelecido também foi de seis meses, todavia, em função da dificuldade no contato para localização e identificação dos profissionais, estendeu-se para 15 meses.

No percurso da produção dos dados, com nutricionistas e com outros profissionais, buscaram-se abarcar as diversas regiões da cidade, de forma a contemplar as diferentes realidades e perspectivas da questão, com a participação de, pelo menos, uma UBS de cada CRS. A coleta de dados foi realizada, por pesquisadores treinados, no local de trabalho dos profissionais nos períodos de julho a dezembro de 2012 e de maio de 2013 a julho de 2014.

Os dados foram produzidos por meio de entrevistas semiestruturadas orientadas por roteiro previamente testado com profissionais da saúde da atenção básica, composto por temas referentes aos grupos, especialmente às estratégias educativas utilizadas e à representação social sobre o processo avaliativo. As respostas foram gravadas, transcritas e, posteriormente, analisadas.

No decorrer da pesquisa, 54 profissionais da saúde concordaram em participar do estudo, sendo 27 nutricionistas de NASF e 27 outros profissionais da atenção básica. Porém, três entrevistas foram realizadas com duplas de profissionais por decisão dos envolvidos. Optou-se pela exclusão desses seis profissionais, para a uniformização da produção e análise dos dados. Dessa forma, foram analisadas 48 entrevistas, 27 com nutricionistas e 21 com outros profissionais.

$\mathrm{Na}$ análise dos dados, utilizou-se, para sistematização e categorização das estratégias educativas, a identificação proposta por Bogdan \& Biklen 12, que procura táticas, métodos, caminhos e técnicas utilizados pelos entrevistados, de forma consciente, para realizar a ação em questão. Após a identificação das categorias, elas foram classificadas em conteúdos de aprendizagem, com base na descrição de Zabala 13 .

Para a identificação das representações sociais dos profissionais sobre o processo avaliativo dos grupos, utilizou-se a técnica do Discurso do Sujeito Coletivo (DSC). Deste modo, em cada depoimento individual, foram selecionadas as expressões-chave, que são os trechos mais significativos das respostas, denominadas de ideias centrais. Em seguida, essas ideias centrais foram organizadas e agrupadas de acordo com elementos da estrutura representacional comum. A partir do agrupamento, foram construídos os discursos-síntese, na primeira pessoa do singular, que são os DSC, para expressar o pensamento da coletividade 14.

Com os DSC formados, as representações sociais foram identificadas à luz da definição apresentada por Jodelet 15. Para a autora, as representações sociais são formas de conhecimento socialmente elaboradas e partilhadas com o objetivo prático de contribuir para a construção de uma realidade comum a um conjunto social. Em sua simbolização, envolvem sensações, ideias, percepções e memórias; e sua interpretação é de caráter construtivo, criativo e autônomo. 
Esta pesquisa foi aprovada pelos Comitês de Ética da Secretaria Municipal de Saúde de São Paulo (parecer no 100/12 e 22/13) e da Faculdade de Saúde Pública da Universidade de São Paulo (parecer no 48307), e seguiu os aspectos éticos da Resolução CNS no 466/2012.

\section{Resultados e discussão}

Fizeram parte do presente estudo 48 profissionais da saúde, sendo 27 nutricionistas, 11 enfermeiros, três médicos, dois auxiliares de enfermagem, dois fonoaudiólogos, um educador físico, um fisioterapeuta e um agente de promoção ambiental, todos atuantes na mediação de grupos que abordam a temática alimentação e nutrição na atenção básica.

Em relação às estratégias educativas utilizadas nos grupos, foram identificadas inicialmente oito categorias: recurso audiovisual, palestra, elementos culinários, dinâmica, roda de conversa, prática corporal, atenção individualizada e abordagens inclusivas. Em seguida, tais categorias foram agrupadas em três conteúdos de aprendizagem, conforme características emergidas durante a análise: conceituais (recurso audiovisual e palestra), que se referem aos conceitos de alimentação e nutrição; procedimentais (elementos culinários, dinâmicas, roda de conversa, prática corporal e atenção individualizada), que são técnicas e métodos aplicados para alcançar finalidades específicas; e atitudinais (abordagens inclusivas), que são as atitudes e os valores envolvidos na ação, apresentados na Tabela 1.

Quanto aos processos avaliativos, encontraram-se oito ideias centrais (Tabela 2) que foram nomeadas de acordo com a estrutura representacional comum dos DSC. As ideias centrais indicam a presença de processos avaliativos direcionados para o indivíduo e outros para o coletivo.

Os dois elementos investigados (Tabelas 1 e 2) são complementares para a prática de grupo. Nesse sentido, as estratégias educativas (Tabela 1) estão apresentadas de forma relacionada com trechos dos DSC derivados das ideias centrais (Tabela 2), nos quais se buscou a identificação das representações sociais dos profissionais da saúde.

Dentre as estratégias educativas referidas pelos profissionais, constatou-se a ampla utilização de recursos audiovisuais, como fôlder, rótulo de alimento, figura, foto e vídeo. Esse achado vai ao encontro do estudo de Araújo et al. 16, que, em duas UBS, verificaram que a maioria dos profissionais utilizava esses materiais nos grupos. Os autores observaram que o uso visava a melhorar a qualidade da explanação da informação abordada pelos profissionais e a propiciar sua compreensão e fixação pelos usuários, isto é, o uso direcionado para a dimensão cognitiva da educação alimentar e nutricional.

De acordo com o marco de referência de educação alimentar e nutricional 7, conduzir um grupo que aborda a alimentação e nutrição inclui, entre as funções do profissional, socializar conhecimentos sobre os alimentos. Nesse sentido, foi verificado o uso de palestra como estratégia educativa que, na maioria das vezes, associa-se à dimensão cognitiva, que tem por objetivo produzir mudanças no comportamento dos participantes como consequência da aquisição de informações. No que se refere ao uso de recurso audiovisual e palestra, os entrevistados mencionaram, em relação ao processo avaliativo, a aquisição de novos conhecimentos, como apresentado no trecho: "Eu vejo mais do conhecimento do que comportamento né, no final do grupo a gente faz uma retomada, o que quê vocês lembram? Então, o conhecimento teórico eu percebo que eles sabem bastante, já se apropriaram e conscientizaram, mas agora na prática são poucas... (DSC - ideias centrais 5).

Notou-se uma analogia entre a avaliação de conhecimento proporcionada pela explanação da informação com o uso de recurso audiovisual ou por meio de palestra, classificados como conteúdos conceituais (Tabela 1). Essa avaliação expressa uma representação social que valoriza o conhecimento científico, legado histórico da educação em saúde 3 e da educação alimentar e nutricional 7 .

O uso dessas estratégias educativas pode ser ampliado para outras intencionalidades. Os recursos audiovisuais podem ser utilizados para promover o diálogo, estimular os sentidos e a criatividade dos participantes, de acordo com a mensagem educativa e as características do grupo 7 . Já a palestra pode ser participativa, quando envolve os participantes, e provocativa, quando os leva a buscarem suas próprias respostas 10. Acentua-se a relevância dessa ampliação, pois Botelho et al. 17 identificaram que a escolha pela palestra é decorrência da rotina de trabalho nas UBS. Apesar de os profissionais reconhecerem que ela não é ideal para a educação em grupo, diante das possibilidades materiais e estruturais, é o recurso mais prático. 


\section{Tabela 1}

Categorias e conteúdos de aprendizagem das estratégias educativas referidas pelos profissionais da saúde de grupos que abordam a alimentação e nutrição. São Paulo, Brasil, 2012-2014.

\begin{tabular}{|c|c|c|c|}
\hline $\begin{array}{l}\text { Categorias das } \\
\text { estratégias }\end{array}$ & $\begin{array}{l}\text { Conteúdos de } \\
\text { aprendizagem }\end{array}$ & Estratégias educativas & $\mathbf{N}$ \\
\hline Recurso audiovisual & Conceituais & $\begin{array}{l}\text { Fôlder, rótulo de alimento, figura, foto, panfleto de supermercado, pirâmide dos } \\
\qquad \text { alimentos, utensílio de cozinha, alimento protótipo, vídeo }\end{array}$ & 24 \\
\hline Palestra & & Palestra temática, aula & 14 \\
\hline Elementos culinários & Procedimentais & $\begin{array}{l}\text { Oficina de papas, oficina de alimentos integrais, oficina de aproveitamento dos } \\
\text { alimentos, oficina de adoçantes, cultivo de hortas }\end{array}$ & 19 \\
\hline Dinâmicas & & $\begin{array}{l}\text { Jogo, brincadeira, dinâmica de apresentação, dinâmica de aquecimento, } \\
\text { pequenos grupos, atividade lúdica }\end{array}$ & 18 \\
\hline Roda de conversa & & Círculo, semicírculo, roda de bate papo & 17 \\
\hline Prática corporal & & Dança circular, alongamento, exercício de fortalecimento, caminhada & 8 \\
\hline Atenção individualizada & & $\begin{array}{c}\text { Diário alimentar, dieta dos pontos, atendimento individual, cardápio } \\
\text { individualizado, dieta individualizada, antropometria }\end{array}$ & 7 \\
\hline Abordagens inclusivas & Atitudinais & $\begin{array}{l}\text { Conhecimento e vivência, ensinar e trocar, problematização, cotidiano, opinião, } \\
\text { vínculo, igualdade, respeito, participação }\end{array}$ & 19 \\
\hline
\end{tabular}

$\mathrm{N}$ : número de profissionais que referiram utilizar as estratégias educativas.

\section{Tabela 2}

Ideias centrais que emergiram nas entrevistas sobre os processos avaliativos de grupos que abordam a alimentação e nutrição. São Paulo, Brasil, 2012-2014.

\begin{tabular}{lc}
\hline Ideias centrais sobre os processos avaliativos dos profissionais da saúde & N \\
\hline 1. Alteração de peso, da pressão, do destro e do perfil lipídico & 25 \\
2. Eu percebo um aumento na assiduidade dos participantes & 18 \\
3. A gente sabe que teve mudança no hábito alimentar & 15 \\
4. A gente avalia vários aspectos da saúde, além da alimentação, e vê resultado no dia a dia deles & 12 \\
5. Eu acho que tem mudança no conhecimento & 11 \\
6. Eles falam das mudanças de casa, da rotina da família & 7 \\
7. Eu acho que o grupo é uma atividade que eles ainda acham muito estranha & 7 \\
8. Eu ainda não tenho um instrumento para avaliar porque o grupo é aberto & 5 \\
\hline
\end{tabular}

$\mathrm{N}$ : número de profissionais que contribuíram para a formação do Discurso do Sujeito Coletivo.

Na educação alimentar e nutricional, além da apreensão de conhecimento, incluem-se também as dimensões afetividade e habilidade, assim, o processo avaliativo dos grupos não deve se restringir à avaliação de conhecimento. Segundo Boog 10 (p. 2015), a prática educativa é realizada "com pessoas, sujeito de sua própria vida e, eventualmente, os resultados alcançados não são exatamente aqueles esperados". Para a autora, quando os objetivos não são atingidos, como observado no DSC supracitado que acena o aumento de conhecimento, mas sem alteração no comportamento de alguns usuários, a avaliação torna-se fundamental, pois irá apontar as direções das reformulações necessárias, seja da informação, seja da estratégia educativa.

Todavia, atenta-se que a educação alimentar e nutricional envolve, além de mudança de comportamento alimentar, comportamentos humanos e relações interpessoais, pois os alimentos atuam como mediadores dos vínculos sociais. Assim, o comportamento alimentar é influenciado por determinantes individuais e coletivos. Naqueles, encontram-se o conhecimento e a percepção sobre a 
alimentação; nesses, os fatores econômicos, sociais e culturais. Assim, o ponto de partida da educação alimentar e nutricional não é a informação, mas a sensibilização dos participantes para ambos os determinantes, que pode ser trabalhada com o uso de estratégias educativas 10.

Outra estratégia identificada foi a utilização de dinâmicas - jogo, brincadeira, atividade lúdica etc. - que contribuem para a aprendizagem, por estimular a compreensão da informação de forma prazerosa. Destaca-se, assim, a importância da ludicidade como recurso educativo, visto que promove a participação e a interação entre os participantes. Por outro lado, sugere-se que o uso deve ser harmônico com a mensagem educativa, não devendo ser utilizado somente para divertir, pois tem por objetivo produzir a construção de novos conhecimentos e novas habilidades 4,18.

A participação e a interação entre os membros dos grupos foram também identificadas no uso de elementos culinários, horta e, principalmente, em oficina culinária. Esta última possibilita a articulação entre a ciência da nutrição e o cotidiano da alimentação, podendo envolver demandas operacionais, como refeições que contemplem os aspectos nutricionais e os princípios do prazer, do custo, da praticidade, do respeito à cultura, da segurança sanitária, entre outros. Além disso, nas vivências culinárias, o fazer, o criar e o degustar preparações estimulam os participantes a ocuparem uma posição de sujeitos em ação 19 .

Ressalta-se que, para o emprego de oficina culinária, é fundamental a disponibilidade de recurso financeiro e espaço adequado, os quais não são frequentemente acessíveis dentro de uma UBS. Logo, as oficinas precisam ser flexíveis, factíveis e adaptadas a cada realidade, sendo que a relação homem-alimento pode ser experimentada em outras atividades, como na realização de lanches, piqueniques, visitas à feira e ao comércio ou, ainda, em preparações simples ou em degustações de receitas práticas 19 .

Uma estratégia distinta apontada pelos profissionais para estimular a participação dos usuários como sujeitos em ação foi associar a nutrição com a prática corporal. Para isso, os profissionais fizeram referência à presença de fisioterapeuta, terapeuta ocupacional ou educador físico. Estudos mostram que a incorporação da prática corporal nos grupos é crescente na atenção básica 3,20, e que ela tem sido primordial para o sucesso de atividades educativas sobre alimentação e nutrição ${ }^{17}$.

Concebe-se que o uso das estratégias educativas - dinâmicas, elementos culinários e prática corporal - classificadas como conteúdos de aprendizagem procedimentais, já que possuem a intencionalidade de, a partir de uma técnica, despertar a participação e a integração dos usuários, agregam a dimensão habilidade da educação alimentar e nutricional. Um trecho que retrata a potencialidade da intervenção grupal com o uso dessas estratégias para a construção de novos hábitos de saúde foi: "A gente avalia vários aspectos da saúde, sono, autoestima, medicação, atividade física e outras. E, pelos relatos, eu percebo mudanças de hábitos devido ao grupo. Era uma vida sedentária e hoje faz atividade física, faz horta em casa, parou de fumar, estima deles elevadíssima e percebo resultado no dia a dia deles, na vida mesmo..." (DSC - ideias centrais 4).

Destaca-se que a associação entre nutrição e atividade física é realizada nas UBS pelo fato de ambos os campos estarem dentro da Política Nacional de Promoção da Saúde 2 como temas prioritários. Por outro lado, essa associação também é encontrada nos grupos que possuem por objetivo a perda de peso corporal dos participantes, já que a demanda para o cuidado de usuários portadores de sobrepeso e obesidade está presente nos grupos das UBS 6 . Argumenta-se que essa demanda pode ocasionar barreiras no desenvolvimento de ações coletivas promotoras de saúde, pois a tendência dos profissionais é individualizar a atenção, método apontado pelos entrevistados nas estratégias educativas (Tabela 1), o que minimiza a potencialidade da intervenção grupal.

A atenção individualizada dentro do grupo sugere estar agregada a um processo avaliativo que tem o foco na mudança do indivíduo, como, por exemplo, o comportamento alimentar, sinalizado pelos entrevistados no trecho: "É a gente tem um indicador que é o questionário de frequência ou recordatório alimentar. Então eu pergunto é uma série de alimentos porque o objetivo principal é a mudança dos hábitos alimentares né e a gente vê que houve mudança mesmo. Tem também as metas que cada pessoa define junto com a gente, é uma meta individual então se eu como muito rápido eu vou soltar o talher no momento da refeição..." (DSC - ideias centrais 3).

Associada ao quadro de sobrepeso e obesidade, constatou-se a demanda para o cuidado de portadores de hipertensão arterial e diabetes mellitus nos grupos mediados pelos profissionais. Para esse processo avaliativo, foi identificado, novamente, o uso de parâmetros individuais: "A gente faz em todos os encontros as aferições antropométricas, vê peso, altura e circunferência abdominal de todos do grupo e vai 
acompanhando a evolução nutricional. (...) E eu peço também exames laboratoriais, controle de pressão e de dentro né, pra gente ir controlando assim. Então tem pessoas que a princípio não perdem peso, mas aí diabetes controlou, a pressão tá controlada, melhorou colesterol, então já é um indicador de uma mudança de comportamento que gerou algum beneficio pra saúde" (DSC - ideias centrais 1 ).

A avaliação antropométrica, a aferição da pressão arterial e da glicemia capilar como um ritual grupal, que tem por função retratar valores e orientações, reafirmando aos participantes a condição de ter a doença, parece ser constante nos grupos das UBS 18,20. Segundo Melo 21, esse monitoramento de saúde-doença traz a ideia de um controle que tem duas concepções: a primeira, sob a ótica do profissional influenciado pela relação de poder perante o outro e, a segunda, uma lógica popular que se volta para as preocupações práticas mobilizadoras dos usuários a realizarem ajustes nas prescrições, na tentativa de equilibrá-las em meio às demandas não biomédicas.

Constatou-se a conexão da avaliação de aquisição de habilidades e comportamentos de saúde com o uso das estratégias educativas - dinâmicas, elementos culinários e prática corporal. Segundo o Marco de Referência de Educação Alimentar e Nutricional para as Politicas Públicas 7, a aquisição de habilidades promove o autocuidado individual, que tem por finalidade o empoderamento dos indivíduos em relação à sua saúde.

Pelos DSC 1, 3 e 4, pode-se observar que os critérios apontados no processo avaliativo possuem o propósito de mensurar resultados objetivos em nível individual. Diante disso, identificou-se a representação social da mudança de comportamento como um resultado esperado pelos profissionais da saúde. Contudo, quando se resgata o objetivo da educação alimentar e nutricional em grupo, constatase que seu processo avaliativo deve incluir critérios que transcendam a objetividade da quantificação e que incorporem elementos da vivência grupal, já que este cuidado é constituído dentro de um espaço social, no qual se busca a grupalidade.

Os critérios objetivos de avaliação, como conhecimento, consumo alimentar, antropometria, controle de pressão arterial e glicemia capilar, são concretizados dentro dos serviços de saúde 22, especialmente nos atendimentos individuais. Entretanto, quando estratégias educativas diferenciadas do atendimento individual são requeridas para estimular participação e integração, considera-se que critérios subjetivos da relação homem-alimento e homem-sociedade sejam incorporados no processo avaliativo.

Ressalta-se que o processo avaliativo de um grupo pode ser entendido por uma análise crítica de sua realização e de seus resultados. Envolve a aplicação de critérios de análise com vistas a conhecer, medir, determinar e julgar a ação, o que estimula a aprendizagem e o aprimoramento dos profissionais da saúde como mediadores de grupos 8,23,24.

A análise crítica de um grupo deve ser feita continuamente, mesmo que de maneira simples, por meio de critérios definidos no planejamento da intervenção educativa 8. Pode-se iniciá-la por critérios quantitativos, já que há dados objetivos disponíveis nas UBS e, posteriormente, incluir os qualitativos por meio da participação dos usuários. A inclusão parte da premissa de que o resultado da ação é um produto da interação entre profissionais da saúde e usuários e que ela favorece a melhoria da qualidade do cuidado, visto que, por meio do diálogo, expressa a subjetividade dos resultados da vivência grupal 25 .

Recomenda-se que os critérios de avaliação sejam definidos com os participantes dos grupos, a partir de seus pontos de vista, mas que os profissionais da saúde incorporem questões do território e do serviço de saúde e/ou informações fundamentadas na literatura científica para fomentar o diálogo 26. Em complemento, destaca-se que o cotidiano dos profissionais e os problemas encontrados nas experiências das ações são fontes de informações, as quais devem ser consideradas no processo avaliativo 7 .

Neste sentido, foi notado que a inclusão dos usuários no processo avaliativo é realizada pelos profissionais por meio de avaliações informais ${ }^{3}$, como os relatos dos participantes: "Na verdade não são mudanças só do paciente, elas conseguiram mudar a casa, a rotina da família, elas trouxeram isso 'ah, eu mudei isso na minha casa, eu usava muito óleo na minha casa, agora eu diminui, minha família não gostou muito, mas eu tô fazendo, ah porque a minha nora ou porque a minha cunhada usa tal produto, mas eu já falei pra ela né" (DSC - ideias centrais 6). Os profissionais apontam que a participação é capaz de gerar confiança e corresponsabilização nos indivíduos, que reafirmam seu empoderamento em outros espaços sociais. Observou-se nesse DSC que há também a representação social voltada para a mudança de comportamento, porém expandida para as famílias. 
Para uma análise participativa, a presença e o envolvimento dos usuários nos grupos são primordiais. Nessa lógica, o processo avaliativo parece não ser algo simples na prática dos profissionais, pois os mesmos referiram que os grupos são "abertos" à população, o que não garante a presença e a análise contínua: "É uma coisa muito difícil. Eu não tenho um instrumento assim pra controlar porque o grupo é aberto, eu já até tentei montar uma ficha, só que aí eu comecei ficar com uma pilha porque às vezes vem um monte de gente, às vezes é pouca, então não tenho como dimensionar" (DSC - ideias centrais 8). Esse DSC traz a representação social da dificuldade de se avaliar uma prática coletiva no contexto da atenção básica, uma vez que, dentre as variáveis, o formato de grupo colocado pelo serviço de saúde marca a irregularidade da presença de usuários, que contribui para a complexidade do planejamento e do desenvolvimento dos grupos 3 .

O resultado dos grupos, sejam "abertos” ou não, depende do grau de envolvimento e de compromisso dos profissionais e dos usuários. Dessa forma, a avaliação participativa por meio da escuta ativa, do reconhecimento dos diferentes saberes, da construção coletiva de novos conhecimentos, da formação de vínculo e das relações horizontais é essencial 7. Caso contrário, os participantes não se sentirão pertencentes ao grupo, o que pode gerar uma desvalorização do atendimento. Isso foi encontrado no presente estudo por meio do seguinte trecho: "Eu acho que eles não valorizam muito, eles acham chato, infelizmente, mas acho que é uma cultura ainda médica, consulta, receita, remédio, dieta milagrosa, então o grupo pra eles ainda é um segundo plano, e é difícil de romper, né. Então muitos pacientes não vêm porque eles acham que não vão ter o atendimento, que não vão sair com que eles precisam..." (DSC - ideias centrais 7). A desvalorização do atendimento em grupo frente ao individual também foi verificada em outros estudos 3,16 que mostram a baixa adesão nos grupos pelos usuários.

Como o atendimento em grupo é uma prática exigida para todos os profissionais da atenção básica, começam a surgir táticas para garantir a presença dos usuários, como a distribuição de brindes, marcação de atendimento individual e entrega de medicamentos 4 , sendo que a última foi explicitada no trecho "...outros vêm porque, por exemplo, no grupo de diabetes eu entrego os insumos, aí eles vêm devido a isso..." (DSC - ideias centrais 7).

Indica-se que a adesão dos grupos está conectada com elementos estruturais e interpessoais. As estruturas das UBS influenciam a presença dos usuários, nas quais algumas limitações para o desenvolvimento são encontradas: ausência de uma área física adequada, falta de investimento em material didático, em recursos de apoio e em modelos educativos 16,27. Isso gera, em algumas situações, o recolhimento de recurso financeiro entre profissionais e usuários para a realização da ação 5. Já os elementos interpessoais envolvem o convite ao grupo e a produção simbólica do primeiro encontro, que marcará o interesse por voltar ou não ao atendimento 18 . Não obstante, adverte-se que o principal influenciador da baixa adesão é o modelo dos grupos que são comumente mediados de modo verticalizado e autoritário por parte dos profissionais 3,17 .

Em alternativa, salienta-se que profissionais que utilizam estratégias educativas voltadas para conteúdos de aprendizagem atitudinais, como as abordagens inclusivas, que podem ser trabalhadas por meio de roda de conversa, favorecem o diálogo e geram resultados promissores, como os descritos nas entrevistas: "O avanço que nós tivemos foi na adesão mesmo dos pacientes. No início era bastante difícil eles virem, eles achavam desnecessário e aí, a partir do momento que eles foram passando, a gente foi conversando, desenvolvendo um vínculo com eles e hoje eles vêm com maior facilidade, dificilmente faltam, então mudou a forma de acesso né. E dá pra perceber que eles estão muito interessados, motivados e que por isso não abandonam o grupo" (DSC - ideias centrais 2).

Compreende-se que as abordagens que buscam a formação de vínculo vão ao encontro dos objetivos da educação alimentar e nutricional, uma vez que envolvem a dimensão afetividade. Ela facilita o alcance das outras dimensões, pois com a inclusão cria-se uma forma de simbolização do cuidado entre os participantes e uma relação de confiança entre usuários e profissionais, o que influencia o comportamento em saúde 4,28.

Nos DSC 7 e 2, observa-se que há a representação social do valor do atendimento coletivo. O primeiro é representado pelas tentativas dos profissionais para que os usuários reconheçam o grupo como um atendimento, e o segundo, pela valorização mediante a formação do vínculo grupal, o que parece garantir a presença dos usuários.

Os DSC, em geral, mostraram que o processo avaliativo dos grupos inclui avaliações informais dos profissionais, identificadas pela manifestação nas expressões “eu acho" e "eu percebo", e também 
dos usuários simbolizadas nos termos "elas trouxeram", "pelos relatos" e "eles acham", que expressam a inclusão desses participantes no processo avaliativo por meio da escuta e/ou da observação realizadas pelos profissionais.

Tal inclusão se aproxima das políticas públicas direcionadoras da atenção básica, as quais enfatizam a promoção da saúde 1,2 e têm a autonomia na área como sua categoria norteadora, conceituada como a capacidade de identificar, questionar e refletir sobre os determinantes da saúde, dentro de um espaço compartilhado de saberes, e de decidir e agir em prol da própria saúde 20,29,30. A afirmação se deve à associação dos seguintes critérios: ampliação de informações sobre saúde, autocuidado individual, participação, integração, corresponsabilização, empoderamento, inclusão e formação de vínculo emergidos nos DSC.

Entretanto, esses critérios apareceram de forma indireta e não sistematizada. Indica-se que esse achado seja consequência de três fatores. O primeiro diz respeito às equipes de ESF inseridas na cidade de São Paulo possuírem como único instrumento avaliativo formalizado e padronizado uma "ficha para registro dos atendimentos de grupo", que contém o número de usuários, profissionais, participantes, modalidade do grupo e temas abordados 3, ou melhor, aspectos objetivos que apontam as ações prestadas à população. O segundo fator é o baixo investimento no planejamento das ações educativas dentro do processo de trabalho das equipes ${ }^{3}$. O terceiro está associado à insuficiência de respaldo teórico da educação alimentar e nutricional para o embasamento das ações, fato que inspirou a elaboração do marco de referência de educação alimentar e nutricional para as políticas públicas em 201228.

Apesar das dificuldades apresentadas para a realização das práticas de grupo, verificou-se neste estudo que elas acontecem e que os profissionais utilizam diversas estratégias educativas, as quais produzem diferentes resultados na vida dos participantes. Entretanto, evidenciam-se obstáculos para a realização de uma análise mais global, dado que as representações sociais dos profissionais estão naturalizadas e normatizadas por um processo de trabalho que é alicerçado em avaliações quantitativas e individualizadas.

Diante disso, reforça-se a importância da superação desses obstáculos para que haja a incorporação de critérios coletivos, conectados com os modos de vida dos participantes, de forma que usuários e profissionais produzam soluções inovadoras para o enfrentamento dos determinantes do comportamento alimentar vivenciados em cada realidade. Por meio dessa produção, concretizam-se os grupos como espaços promotores de saúde e do direito humano à alimentação adequada, o que tem sido um dos desafios da área da saúde e da segurança alimentar e nutricional 6,28.

\section{Conclusão}

O estudo analisou as representações sociais dos profissionais da atenção básica sobre processos avaliativos de grupos que abordam a alimentação e nutrição, e descreveu as estratégias educativas utilizadas.

Foram identificadas nas estratégias educativas os três conteúdos de aprendizagem: conceituais, procedimentais e atitudinais. Isso mostra que os profissionais estão atentos aos conceitos, às técnicas e aos métodos de desenvolvimento de grupos, mas também às formas de abordagem para essa aplicação. As abordagens inclusivas identificadas estão de acordo com o referencial teórico da educação alimentar e nutricional adotado no estudo, e podem ser fortalecidas, posto que contribuem para cuidado integral.

Já no processo avaliativo, verificaram-se nos DSC quatro representações sociais: valorização do conhecimento, mudança de comportamento de saúde, dificuldade de se avaliar e valor do atendimento. Elas evidenciam a existência de um conflito entre produzir a ação, dentro de um processo de trabalho enraizado no histórico da educação em saúde, e tentativas de promover saúde, por meio da avaliação participativa. Nessas tentativas, constatou-se a presença implícita da autonomia em saúde, porém voltada, predominantemente, para os critérios individuais. Não obstante, pode-se considerar um avanço, dado que, no âmbito institucional do serviço, sobressaem-se critérios quantitativos de avaliação, o que reforça uma análise superficial e não sistematizada dos espaços coletivos.

Recomenda-se aos profissionais, mediadores de grupos de alimentação e nutrição, que produzam critérios de avaliação de seus próprios grupos, pautados no marco de referência de educação alimen- 
tar e nutricional para as políticas públicas, para dar visibilidade a um trabalho que já é realizado e, assim, possibilitar que esses critérios sejam reconhecidos e incorporados à rotina dos serviços e da gestão em saúde. Propõe-se que os critérios individuais e coletivos sejam avaliados conjuntamente, dado que o grupo produz resultados em níveis individuais e coletivos. A construção de critérios individuais pode considerar o conhecimento, a construção de novos conhecimentos, a aquisição de novas habilidades, a mudança de atitudes e valores, a autonomia em saúde e, também, o estado nutricional e de saúde. Em alternativa, os critérios coletivos podem incluir comunicação, participação, integração, formação de vínculo, corresponsabilização e empoderamento comunitário.

Estratégias educativas e processos avaliativos que coloquem os diferentes atores em uma posição de reciprocidade sem desfigurar o papel de educador dos profissionais são oportunas e essenciais, portanto, ressalta-se a necessidade de incentivo financeiro e de educação permanente no planejamento dos grupos da atenção básica.

\section{Colaboradores}

K. R. R. Vincha participou da análise dos dados, da interpretação dos resultados, da redação do artigo e da aprovação final da versão a ser publicada. V. L. Vieira participou da análise dos dados, da interpretação dos resultados e da redação do artigo. L. D. S. Guerra, F. C. Botelho e A. Pava-Cárdenas participaram da coleta de dados, da redação do artigo e da aprovação final da versão a ser publicada. A. M. Cervato-Mancuso participou da interpretação dos resultados, da redação do artigo e da aprovação final da versão a ser publicada.

\section{Agradecimentos}

Ao apoio financeiro do Conselho Nacional de Desenvolvimento Científico e Tecnológico (CNPq, 481712/2011-9) e da Fundação de Amparo à Pesquisa do Estado de São Paulo (FAPESP, 022642/2012).

\section{Referências}

1. Departamento de Atenção Básica, Secretaria de Atenção à Saúde, Ministério da Saúde. Política Nacional de Atenção Básica. Brasília: Ministério da Saúde; 2012. (Série E. Legislação em Saúde).

2. Secretaria de Atenção à Saúde, Secretaria de Vigilância em Saúde, Ministério da Saúde. Política Nacional de Promoção da Saúde PnaPS: revisão da Portaria MS/GM no 687, de 30 de março de 2006. Brasília: Ministério da Saúde; 2014.

3. Ferreira-Neto JL, Kind L. Promoção da saúde: práticas grupais na Estratégia Saúde da Família. Belo Horizonte: Fapeming; 2011.

4. Almeida ER, Moutinho CB, Leite MTDS. A prática da educação em saúde na percepção dos usuários hipertensos e diabéticos. Saúde Debate 2014; 38:328-37.

5. Vasconcelos ACCP, Magalhães R. Práticas educativas em segurança alimentar e nutricional: reflexões a partir da experiência da Estratégia Saúde da Família em João Pessoa, PB, Brasil. Interface (Botucatu, Online) 2016; 20:99-110.

6. Vincha KRR, Cárdenas AP, Cervato-Mancuso AM, Vieira VL. Grupos de educação nutricional em dois contextos da América Latina: São Paulo e Bogotá. Interface (Botucatu, Online) 2014; 18:507-20. 
7. Secretaria Nacional de Segurança Alimentar e Nutricional, Ministério de Desenvolvimento Social e Combate à Fome. Marco de referência de educação alimentar e nutricional para as políticas públicas. Brasília: Ministério de Desenvolvimento Social e Combate à Fome; 2012.

8. Cervato-Mancuso AM. Elaboração de um programa de educação nutricional. In: Diez-Garcia RW, Cervato-Mancuso AM, organizadores. Mudanças alimentares e educação nutricional. Rio de Janeiro: Editora Guanabara Koogan; 2011. p. 187-97.

9. Cardoso AVL, Chain APN, Mendes RIP, Ferreira e Ferreira E, Vargas AMD, Martins AMEBL, et al. Avaliação da gestão da Estratégia Saúde da Família por meio do instrumento avaliação para melhoria da qualidade em municípios de Minas Gerais, Brasil. Ciênc Saúde Coletiva 2015; 20:1267-84.

10. Boog MCF. Educação em nutrição: integrando experiências. Campinas: Komedi; 2013.

11. Biernacki P, Waldford D. Snowball sampling: problems and techniques of chain referral sampling. Sociol Methods Res 1981; 10:141-63.

12. Bogdan R, Biklen S. Investigação qualitativa em educação: uma introdução à teoria e aos métodos. Porto: Porto Editora; 1994.

13. Zabala A. A prática educativa: como ensinar. Porto Alegre: Editora Artmed; 1998.

14. Lefèvre F, Lefèvre AMC, Teixeira JJV. O discurso do sujeito coletivo: uma abordagem em pesquisa qualitativa. Caxias do Sul: Educs; 2003.

15. Jodelet D. As representações sociais: um domínio em expansão. In: Jodelet D, organizador. As representações sociais. Rio de Janeiro: EdUERJ; 2001. p. 17-44.

16. Araújo V, Dias M, Bustorff L. A instrumentalização da educação em saúde na atenção básica. Revista de Enfermagem Referência 2011; serIII:7-17.

17. Botelho FC, Guerra LDS, Pava-Cárdenas A, Cervato-Mancuso AM. Estratégias pedagógicas em grupos com o tema alimentação e nutrição: os bastidores do processo de escolha. Ciênc Saúde Coletiva 2016; 21:1889-98.

18. Nunes JM, Oliveira EN, Machado MFAS, Costa PNP, Vieira NFC. A participação de agentes comunitários de saúde em grupo de educação em saúde. Rev RENE 2012; 13:1084-91.
19. Rotenberg S, Marcolan S, Tavares EL, Castro IRR. Oficinas culinárias na promoção da saúde. In: Diez-Garcia RW, Cervato-Mancuso AM, organizadores. Mudanças alimentares e educação nutricional. Rio de Janeiro: Editora Guanabara Koogan; 2011. p. 327-39.

20. Carneiro ACLL, Souza V, Godinho LK, Faria ICMF, Silva KL, Gazzinelli MF. Educação para a promoção da saúde no contexto da atenção primária. Rev Panam Salud Pública 2012; 31:115-20.

21. Melo LP. Análise biopolítica do discurso oficial sobre educação em saúde para pacientes diabéticos no Brasil. Saúde Soc 2013; 22:1216-25.

22. Cervato-Mancuso AM, Vincha KRR, Santiago DA. Educação Alimentar e Nutricional como prática de intervenção: reflexão e possibilidades de fortalecimento. Physis (Rio J.) 2016; 26:225-49.

23. Bosi MLM, Uchimura KY. Avaliação qualitativa de programas de saúde: contribuições para propostas metodológicas e centradas na integralidade e na humanização. In: Bosi MLM, Mercado FJ, organizadores. Avaliação qualitativa de programas de saúde: enfoques emergentes. Petrópolis: Editora Vozes; 2006. p. 87-117.

24. Sobral NAT, Santos SMC. Proposta metodológica para avaliação de formação em alimentação saudável. Rev Nutr 2010; 23:399-415.

25. Tanaka OY. Avaliação da atenção básica em saúde: uma nova proposta. Saúde Soc 2011; 20:927-34.

26. Furtado JP. Um método construtivista para a avaliação em saúde. Ciênc Saúde Coletiva 2001; 6:165-81.

27. Afonso RR, Lemos A. Adesão nos grupos educativos em contracepção em uma área programática do Rio de Janeiro. Rev Enferm Cent-Oeste Min 2011; 1:238-47.

28. Cervato-Mancuso AM, Fiore EG, Redolfi SCS. Guia de segurança alimentar e nutricional. Barueri: Edições Manole; 2015.

29. Haeser LM, Büchele F, Brzozowski FS. Considerações sobre a autonomia e a promoção da saúde. Physis (Rio J.) 2012; 22:605-20.

30. Fleury-Teixeira P, Vaz FAC, Campos FCC, Álvares J, Aguiar RAT, Oliveira VA. Autonomia como categoria central no conceito de promoção de saúde. Ciênc Saúde Coletiva 2008; 13 Suppl 2:2115-22. 


\section{Abstract}

The study analyzed the social representations of primary health care professionals on evaluative processes of groups that work with food and nutrition, and described the educational strategies used in this care. This was a qualitative study from 2012 to 2014 in the city of São Paulo, Brazil, in which 48 interviews were analyzed. In the analysis of the interviews, for classification of the educational strategies in learning categories and contents, Bogdan es Biklen and Zabala were used, respectively. The evaluative processes used the collective subject discourse technique, based on Jodelet's social representations. Three learning contents were found in the educational strategies and four social representations of the evaluative processes which combined to reveal the presence of a conflict by a practice directed by the work process to quantitative and individual evaluative criteria and a health-promoting practice that used inclusive approaches and participant evaluation. In this practice, the study implicitly identified the presence of autonomy in health. The study revealed the need to acknowledge and systematize group planning as an educational tool that qualifies and empowers comprehensive care.

Primary Health Care; Health Education; Food and Nutrition Education; Process Assessment (Health Care)

\section{Resumen}

El estudio analizó las representaciones sociales de los profesionales de la salud de la atención primaria de salud, sobre los procesos evaluativos de grupos que abordan alimentación y nutrición, y describió las estrategias educativas usadas al respecto. Se trata de una investigación cualitativa, llevada a cabo en la ciudad de São Paulo, Brasil, entre 2012 y 2014, en la que fueron analizadas 48 entrevistas. En el análisis de las entrevistas, para la clasificación de las estrategias educativas en categorías y contenidos de aprendizaje, se siguió la propuesta de Bogdan es Biklen y Zabala, respectivamente. Para los procesos evaluativos, se utilizó la técnica del Discurso del Sujeto Colectivo, fundamentada en las representaciones sociales de Jodelet. Fueron verificados tres contenidos de aprendizaje de estrategias educativas y cuatro representaciones sociales de los procesos evaluativos que, asociados, evidenciaron la presencia de un conflicto entre por un lado, una práctica dirigida por el proceso de trabajo para criterios de evaluación cuantitativos $e$ individuales $y$, por otro lado, una práctica promotora de la salud, mediante el uso de abordajes inclusivos y de evaluación participativa. En esta práctica, se identificó, implícitamente, la presencia de la autonomía en salud. Además, se evidenció la necesidad de reconocer y sistematizar la planificación de los grupos como una herramienta educativa que cualifica y potencia el cuidado para una atención integral.

Atención Primaria de Salud; Educación en Salud; Educación Alimentaria y Nutricional; Evaluación de Proceso (Atención de Salud)
Recebido em 07/Mar/2016

Versão final reapresentada em 11/Out/2016 Aprovado em 04/Nov/2016 